\title{
Resistance to ventricular fibrillation predicted by the QRS/QTc - Ratio in an intact rat model of hypothermia/rewarming
}

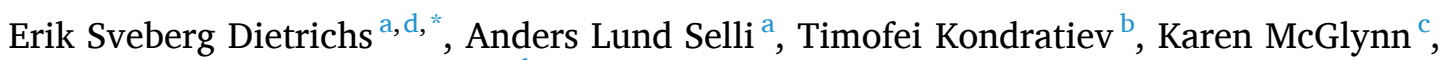 \\ Godfrey Smith ${ }^{c}$, Torkjel Tveita ${ }^{\text {b, }}$ \\ ${ }^{a}$ Experimental and Clinical Pharmacology, Department of Medical Biology, UiT, The Arctic University of Norway, 9037, Troms $\phi$, Norway \\ ${ }^{\mathrm{b}}$ Anesthesia and Critical Care Research Group, Department of Clinical Medicine, UiT, The Arctic University of Norway, 9037, Troms $\phi$, Norway \\ ${ }^{\mathrm{c}}$ Institute of Cardiovascular \& Medical Sciences, University of Glasgow, UK \\ ${ }^{\mathrm{d}}$ Division of Diagnostic Services, University Hospital of Northern Norway, Troms $\phi$, Norway \\ ${ }^{\mathrm{e}}$ Division of Surgical Medicine and Intensive Care, University Hospital of North Norway, 9038, Tromsø, Norway
}

\section{A R T I C L E I N F O}

\section{Keywords:}

Accidental hypothermia

Therapeutic hypothermia

ECG

QRS/QTc

Arrhythmia

Ventricular fibrillation

\begin{abstract}
A B S T R A C T
Accidental hypothermia is associated with increased risk for arrhythmias. QRS/QTc is proposed as an ECGmarker, where decreasing values predict hypothermia-induced ventricular arrhythmias. If reliable it should also predict nonappearance of arrhythmias, observed in species like rat that regularly tolerate prolonged hypothermia.

A rat model designed for studying cardiovascular function during cooling, hypothermia and subsequent rewarming was chosen due to species-dependent resistance to ventricular arrhythmias. ECG was recorded throughout the protocol.

No ventricular arrhythmias occurred during experiments. QRS/QTc increased throughout the cooling period and remained above normothermic baseline until rewarmed.

Different from the high incidence of hypothermia-induced ventricular arrhythmias in accidental hypothermia patients, where QRS/QTc ratio is decreased in moderate hypothermia; hypothermia and rewarming of rats is not associated with increased risk for ventricular fibrillation. This resistance to lethal hypothermia-induced arrhythmias was predicted by QRS/QTc.
\end{abstract}

\section{Introduction}

Hypothermia is defined as a reduction of core-temperature to below $35^{\circ} \mathrm{C}[17]$. The neuroprotective effect of hypothermia is undisputed, but it is questioned whether therapeutic hypothermia, with core temperature reduction below $35{ }^{\circ} \mathrm{C}$, should be initiated in comatose survivors of cardiac arrest [3]. Current advice is to apply targeted temperature management $\left(36-32{ }^{\circ} \mathrm{C}\right)$ for neuroprotection in these patients [17]. During surgical procedures like reconstructive cardiac surgery, the neuroprotective effect of cooling is evident and core temperature is occasionally reduced to below $20{ }^{\circ} \mathrm{C}$ [3]. Similar neuroprotection is obvious in victims of accidental hypothermia, where survival after several hours of cardiac arrest is reported after core-temperature reduction down to $13.7^{\circ} \mathrm{C}[10]$.
Neuroprotective effects of hypothermia are present at temperatures where increased risk for arrhythmias and cardiac arrest occur, a wellknown complication of hypothermia [8]. In humans, changes in electrocardiography (ECG) recordings, including prolongation of QT-interval and sinus bradycardia are common at core-temperatures above $30{ }^{\circ} \mathrm{C}$, during use of mild therapeutic hypothermia. Further cooling will increase the risk for induced arrhythmias, including nodal rhythms, ventricular extrasystoles, atrio-ventricular blocks and ventricular fibrillation (VF) [8]. The underlying mechanisms causing pro-arrhythmic activity in hypothermia, however, are not well known. This lack of evidence-based knowledge is reflected by guidelines for treating victims of accidental hypothermia, where recommendations for prevention and treatment of hypothermia-induced arrhythmias are missing [23].

Abbreviations: VF, Ventricular fibrillation; VT, Ventricular tachycardia; HR, Heart rate; HCA, Hypothermic cardiac arrest; ECG, Electrocardiography; APD, Action potential duration.

* Corresponding author. Experimental and Clinical Pharmacology, Institute of Medical Biology, UiT, The Arctic University of Norway, 9037, Troms $\varnothing$, Norway.

E-mail address: erik.sveberg.dietrichs@uit.no (E.S. Dietrichs). 
In a series of recent preclinical experiments, we have investigated the pathophysiology of hypothermia-induced VF and cardiac arrest (HCA) [6-8]. Our findings show that moderate hypothermia $\left(32-28{ }^{\circ} \mathrm{C}\right)$ is pro-arrhythmic [6-8]. An underlying depolarisation-repolarisation mismatch is apparent in rabbit hearts [6], a species with similar cardiac electrophysiology to humans. This mismatch was prominent at $31{ }^{\circ} \mathrm{C}$ and was associated with a doubled risk for inducing VF [6]. Further temperature reduction, towards $20{ }^{\circ} \mathrm{C}$ and below, appears anti-arrhythmic in these experiments [6-8]. Further, we found that a novel ECG-marker (QRS/QTc) could predict risk for VF in hypothermic rabbit hearts and have advocated that it shows potential in hypothermic patients [7]. The utility of this marker was dependent on heart rate adjustment to correlate with HCA-risk, different from the iCEB-marker for Torsades de Pointes [15].

In contrast to rabbits that have cardiac electrophysiology resembling humans and are at risk for developing hypothermia-induced VF [6-8, 12], rats do not regularly develop VF, even during several hours of exposure to core temperatures between 15 and $13{ }^{\circ} \mathrm{C}[4,5,24]$. This arrhythmia-resistance is interesting as it allows us to study development of QRS/QTc-values during cooling to severe hypothermia, when VF-risk is low. Accordingly, we decided to investigate cardiac electrophysiology using our well-established rat model of severe hypothermia $\left(15^{\circ} \mathrm{C}\right)$, to assess whether QRS/QTc could predict occurrence or absence of spontaneous, hypothermia-induced ventricular arrhythmias.

\section{Methods}

\subsection{Ethics approval and consent to participate}

Male Wistar rats $(\mathrm{n}=6)$ weighing $270-346 \mathrm{~g}$ were used in the experiment. The rats (Charles River, Germany) had a microbiological status according to the recommendation of the Federation of European Laboratory Animal Science Associations. Housing was provided in accordance with guidelines for accommodation and care of animals (article 5 of European Convention for the Protection of Vertebrate Animals used for Experimental and Other Scientific Purposes, Strasbourg, 18.III.1986). The experimental protocol was approved by the Norwegian Animal Research Authority and conducted accordingly.

\subsection{Housing}

The animals were quarantined for 1 week on arrival and housed in a conventional SPF facility in Eurostandard Type IV cages (2 rats per cage) with wood shavings as bedding (Aspen Bedding, Tapvei, Estonia). Housing conditions were controlled with temperature and humidity maintained at $21 \pm 1{ }^{\circ} \mathrm{C}$ and $55 \pm 5 \%$, respectively, and the lighting on a $12 \mathrm{~h}$ light and $12 \mathrm{~h}$ dark cycle. The rats were fed ad libitum with a conventional rodent maintenance diet (RM1-E, Special Diet Service, UK) and tap water. Polycarbonate Rat Tunnels and aspen gnawing bricks (both from Scanbur, Denmark) were provided as environmental enrichment. The animals were allowed to acclimatize for a period of one week after entering the facility and were under daily health checks during the housing period prior to the terminal experiment.

\subsection{Anesthesia}

All experiments were started by introducing anesthesia intraperitoneally; $55 \mathrm{mg} / \mathrm{kg}$ pentobarbital sodium and $50 \mu \mathrm{g} / \mathrm{kg}$ fentanyl, followed by a continuous infusion of $7.5 \mathrm{mg} / \mathrm{kg} /$ hour pentobarbital sodium and $50 \mu \mathrm{g} / \mathrm{kg} /$ hour fentanyl through an intravenous line in the right jugular vein, extended to the right auricle. The infusion was maintained at all hours in normothermic animals. Infusion in hypothermic animals was terminated at $30{ }^{\circ} \mathrm{C}$ during cooling and restarted at the same temperature during rewarming, due to hypothermia-induced anesthesia and reduced drug metabolism. During the experiments, toe pinching was used continuously, and blood gasses, respiratory rate and heart rate monitored closely to ensure that the animals were provided with sufficient anesthesia and analgesia. If any signs of stress were found, anesthesia and analgesia was increased through the intravenous line. After rewarming, animals were euthanized by a bolus dose of pentobarbital sodium and fentanyl through the intravenous line.

\subsection{Respiratory support}

Animals were placed on the operating table in a supine position. The trachea was opened, and a tracheal tube inserted. All animals had spontaneous and sufficient ventilation at core temperatures $>20{ }^{\circ} \mathrm{C}$. Below $20^{\circ} \mathrm{C}$, ventilation was achieved by a volume-controlled New England rodent ventilator, model 141 (New England Instruments, Massachusetts, USA), using room air. Normoventilation was achieved through adjusting ventilation in accordance with blood gas analyzes from an ABL 800 blood gas analyzer (Radiometer, Denmark). During controlled ventilation, the alpha-stat strategy was followed.

Electrocardiographic variables were recorded using a 3-channel Fe136 Animal Bio Amp device (ADInstruments, Dunedin, New Zealand).

\subsection{Core cooling and rewarming}

Animals were cooled and rewarmed using RTE-110 thermo stated water bath (Neslab Instruments, New Hampshire, USA) by circulating cold or warm water through a U-shaped polyethylene tube, which was inserted gently into the lower bowel to avoid harm to the intestine. In addition, the double-layered operating table made of hollow aluminum was circulated by temperature-adjusted water. Core temperature was continuously monitored using a thermocouple wire positioned in the lowest part of esophagus, connected to a Thermalert Th-5 thermocouple controller (Bailey Instruments, UK). Cooling and rewarming each lasted $115 \pm 48 \mathrm{~min}$ and $98 \pm 42 \mathrm{~min}$ respectively, and the stable hypothermic period $\left(15{ }^{\circ} \mathrm{C}\right)$ lasted $4 \mathrm{~h}$. Measurements of recorded variables were performed at set temperatures $\left(37,32,28,24,20\right.$ and $15{ }^{\circ} \mathrm{C}$ ) during cooling and rewarming. During stable hypothermia, variables were recorded every hour. The rate of core rewarming was based on clinical practice in our university hospital, where fast rewarming has proven successful in hypothermic patients after nearly $7 \mathrm{~h}$ of hypothermic cardiac arrest.

\subsection{Electrocardiographic measurements}

Electrocardiographic variables were recorded using a 3-channel Fe136 Animal Bio Amp device (ADInstruments, Dunedin, New Zealand). Left ventricular systolic and diastolic duration was obtained using a SPR-838 Millar pressure-volume conductance catheter (Millar Instruments Inc., Texas, USA). The miniaturized 2.0 french pressure-volume conductance catheter allows assessment of in vivo LV mechanical function in rats (1). All variables in this study were calculated using different modules in the LabChart Pro v8.1.3 software package (ADInstruments, Dunedin, New Zealand).

The present model has been used in numerous studies in our lab, and the stability of physiological parameters during $4-5 \mathrm{~h}$ of normothermic control conditions have been properly documented [4,5].

\subsection{Statistics}

Sample size calculations were performed in MedCalc 7.2 (MedCalc Software, Mariakerke, Belgium). Animals served as their own controls, with continuous monitoring of cardiac electrophysiology. Changes occurring during cooling, stable hypothermia and rewarming respectively were analyzed by One-way repeated measures ANOVA. Dunnett's method was used to compare values during cooling with prehypothermic baseline, hypothermic values with hypothermic baseline and changes occurring during rewarming with values at the end of the stable hypothermia period. To assess differences between pre- 
hypothermic and rewarmed values at $37{ }^{\circ} \mathrm{C}$, a paired $t$-test was used. Results are presented as mean \pm SEM. Differences were considered significant at $\mathrm{p}<0.05$.

\section{Results}

No ventricular arrhythmias were observed at any temperatures during cooling, stable hypothermia, or rewarming in this experiment.

\subsection{Comparison of variables at pre-hypothermic baseline vs. after rewarming $\left(37^{\circ} \mathrm{C}\right)$ (Table 1$)$}

Compared to pre-hypothermic values at $37{ }^{\circ} \mathrm{C}$, no changes in heart rate (HR) or ECG-characteristics, including QRS/QTc, were found at $37^{\circ} \mathrm{C}$ after rewarming.

\subsection{Cooling to $15{ }^{\circ} \mathrm{C}$ (Figs. 1-3)}

During cooling to $15^{\circ} \mathrm{C}$ a significant prolongation of all aspects of the ECG recording occurred, when compared to baseline values at $37^{\circ} \mathrm{C}$. At temperatures including $28{ }^{\circ} \mathrm{C}$ and below, the QT-interval was significantly increased $(0.07 \pm 0.01 \mathrm{sec}$ vs. $0.05 \pm 0.01 \mathrm{sec}, \mathrm{p}<0.05)$. RR $(0.23$ $\pm 0.04 \mathrm{sec}$ vs. $0.12 \pm 0.04 \mathrm{sec}, \mathrm{p}<0.05)$, PR $(0,07 \pm 0.01 \mathrm{sec}$ vs. $0.05 \pm$ $0.01 \mathrm{sec}, \mathrm{p}<0.05)$, and QRS (0.04 $\pm 0.01 \mathrm{sec}$ vs. $0,03 \pm 0.01 \mathrm{sec}, \mathrm{p}<$ 0.05 ) intervals where prolonged after cooling to $24{ }^{\circ} \mathrm{C}$, while QR time $(0,05 \pm 0.02 \mathrm{sec}$ vs. $0,01 \pm 0.00 \mathrm{sec}, \mathrm{p}<0.05)$ was increased only after cooling to $20{ }^{\circ} \mathrm{C}$. Consistent with prolongation of the RR interval, HR (272 $\pm 22 \mathrm{bpm}$ vs. $448 \pm 47 \mathrm{bpm}, \mathrm{p}<0.05)$ was significantly reduced after cooling to $28^{\circ} \mathrm{C}$.

There was no sign of an initial reduction of QRS/QTc values during moderate hypothermia, as they showed a steady increase throughout the entire cooling period, with significantly elevated values after cooling to $24{ }^{\circ} \mathrm{C}(0.35 \pm 0.06 \mathrm{~s} / \mathrm{s}$ vs. $0.22 \pm 0.05 \mathrm{~s} / \mathrm{s}, \mathrm{p}<0.05)$..

\subsection{Stable hypothermia $\left(4 \mathrm{~h}\right.$, at $\left.15{ }^{\circ} \mathrm{C}\right)$ (Figs. 1-3)}

During stable hypothermia, all variables in the ECG-recording remained stable except for the PR interval $(0,19 \pm 0.05 \mathrm{sec}$ vs. $0.16 \pm$ $0.06 \mathrm{sec}, \mathrm{p}<0.05$ ) that increased during the hypothermic period. HR remained stable.

QRS/QTc values remained statistically unchanged during the $4 \mathrm{~h}$ hypothermia period.

\subsection{Rewarming to $37^{\circ} \mathrm{C}$ (Figs. 1-3)}

During rewarming from $15^{\circ} \mathrm{C}$ to $37^{\circ} \mathrm{C}$ a significant shortening of the QR time $(0,01 \pm 0.00$ sec vs. $0,05 \pm 0.04 \mathrm{sec}, \mathrm{p}<0.05)$, QT $(0,05 \pm$ $0.01 \mathrm{sec}$ vs. $0,14 \pm 0.07 \mathrm{sec}, \mathrm{p}<0.05), \mathrm{QRS}(0,03 \pm 0.01 \mathrm{sec}$ vs. $0,08 \pm$ $0.05 \mathrm{sec}, \mathrm{p}<0.05)$ and PR $(0,04 \pm 0.00$ sec vs. $0,11 \pm 0.02 \mathrm{sec}, \mathrm{p}<0.05)$ intervals were observed at $24{ }^{\circ} \mathrm{C}$, while the RR interval $(0,69 \pm 0.23 \mathrm{sec}$ vs. $1,10 \pm 0.15 \mathrm{sec}, \mathrm{p}<0.05$ ) was reduced during rewarming to $20^{\circ} \mathrm{C}$. HR $(165 \pm 34 \mathrm{bpm}$ vs. $57 \pm 9 \mathrm{bpm}, \mathrm{p}<0.05)$ increased significantly

Table 1

Electrocardiographic variables before cooling $\left(37^{\circ} \mathrm{C}\right)$ and after rewarming from $4 \mathrm{~h}$ of stable hypothermia $\left(15^{\circ} \mathrm{C}\right)$. * Significant $(\mathrm{p}<0.05)$ difference from precooling $\left(37^{\circ} \mathrm{C}\right)$ baseline.

\begin{tabular}{llll}
\hline ECG and Cycle duration & Pre-cooling $37{ }^{\circ} \mathrm{C}$ & Rewarmed $37{ }^{\circ} \mathrm{C}$ & P-value \\
\hline QRS/QTc (sec/sec) & $0.224 \pm 0.020$ & $0.203 \pm 0.020$ & 0,34 \\
QT interval (sec) & $0.045 \pm 0.003$ & $0.046 \pm 0.004$ & 0,88 \\
QTc interval (sec) & $0.136 \pm 0.002$ & $0.137 \pm 0.001$ & 0,97 \\
QR time (sec) & $0.010 \pm 0.001$ & $0.011 \pm 0.001$ & 0,47 \\
QRS interval (sec) & $0.029 \pm 0.002$ & $0.028 \pm 0.003$ & 0,61 \\
PR interval (sec) & $0.046 \pm 0.004$ & $0.037 \pm 0.002$ & 0,60 \\
RR interval (sec) & $0.123 \pm 0.020$ & $0.111 \pm 0.010$ & 0,58 \\
Heart rate (beats/min) & $448 \pm 13.0$ & $446 \pm 18.5$ & 0,99 \\
\hline
\end{tabular}

during rewarming to $24^{\circ} \mathrm{C}$.

During rewarming, the QRS/QTc $(0.49 \pm 0.15 \mathrm{~s} / \mathrm{s}$ vs. $0.62 \pm 0.09 \mathrm{~s} / \mathrm{s}$, $\mathrm{p}<0.05)$ values decreased rapidly with significantly reduced values already after rewarming to $20^{\circ} \mathrm{C}$. Similar to cooling, rewarming did not give a reduction of QRS/QTc to below baseline, as values remained higher than at $37^{\circ} \mathrm{C}$ until animals were rewarmed.

\section{Discussion}

In the present intact rat study, we find that despite the presence of significant changes in cardiac electrophysiology induced by severe hypothermia and rewarming, no ventricular arrhythmias took place. All electrophysiological parameters were however normalised after rewarming. As predicted by the QRS/QTc ratio values, which remained higher than at normothermia throughout the protocol, we found that neither cooling, prolonged hypothermia, nor rewarming created substrate(s) for lethal ventricular arrhythmias in our rat model.

In humans, spontaneous ventricular arrhythmias are common in victims of accidental hypothermia [8]. Accordingly, it is interesting to assess risk for spontaneous $\mathrm{VF}$, considering the electrophysiological differential effects of hypothermia between humans and rats. The hypothermia-induced electrophysiological changes, in the presence of intact sinus rhythm, as observed in the present experiment, therefore gives valuable information of potential translational value. Background pathophysiologic mechanisms for hypothermia-induced VF and cardiac arrest in patients has been largely unknown [8]. However, recent efforts with translational research into this topic have given possible groundbreaking results [7]. In rabbit, both increased ventricular divergence [12] and heterogenic effect of hypothermia on transmural and longitudinal conduction [6] has been documented at $30{ }^{\circ} \mathrm{C}$, with increased risk for developing VF. Cooling to $30{ }^{\circ} \mathrm{C}$ also enhanced dispersion of epicardial action potential duration (APD), wavebreaks and re-entry, associated with increased vulnerability to pacing-induced VF [13]. In pigs cooled to $30{ }^{\circ} \mathrm{C}$, VF threshold is reduced by $72 \%$ [25]. This is also found in dogs at $25^{\circ} \mathrm{C}$, where VF threshold was reduced compared to at $37{ }^{\circ} \mathrm{C}$ [1]. Cardiac vulnerability, however, does seem to be promoted further by rewarming. In canine wedge preparations cooled to $26^{\circ} \mathrm{C}, \mathrm{VF}$ and ventricular tachycardia (VT) occurred more frequently during rewarming than during cooling [19]. In a similar model, hypothermia $\left(32{ }^{\circ} \mathrm{C}\right)$ caused local re-excitation and development of polymorphic VT/VF [11]. In two recent publications, we reported that the QRS/QTc ratio could be used as a highly predictable marker of VF threshold in rabbits $[6,7]$.

Ventricular ectopic activity is documented being increased in patients treated with therapeutic hypothermia [22], in addition to the occurrence of frequent non-sustained VT [20]. However, most studies report that sustained ventricular arrhythmias are uncommon [8], with some exceptions as by Mirzoyev et al. who documented polymorphic VT in $11.7 \%$ of therapeutic hypothermia patients [16]. Most cases with VT occurred at a core temperature around $34.7^{\circ} \mathrm{C}$, and defibrillation was necessary in most of these patients. Patients with polymorphic VT were hypokalemic and had significantly prolonged QTc interval [16]. Risk for $\mathrm{VF}$ is dependent of severity of hypothermia and pose a big challenge during rewarming. Of 19 accidental hypothermia patients admitted, with core temperatures between $17{ }^{\circ} \mathrm{C}-29{ }^{\circ} \mathrm{C}$, seven were in VF, while two presented with asystole [2]. In a Japanese study of 60 patients, no patients with a core temperature above $26{ }^{\circ} \mathrm{C}$ developed VF [18]. Recently we published a report indicating that the QRS/QTc interval could be used as a biomarker of risk for hypothermia-induced VF in such patients [7].

In the present study, we show that rats are largely resistant to spontaneous ventricular arrhythmia, induced by hypothermia. Still, rats are vulnerable to pacing-induced arrhythmias and several studies of normothermic cardiopulmonary resuscitation are carried out in wellestablished rat models of VF $[9,14]$. The relative different electrophysiological effects when cooling rats, compared to hypothermic patients 

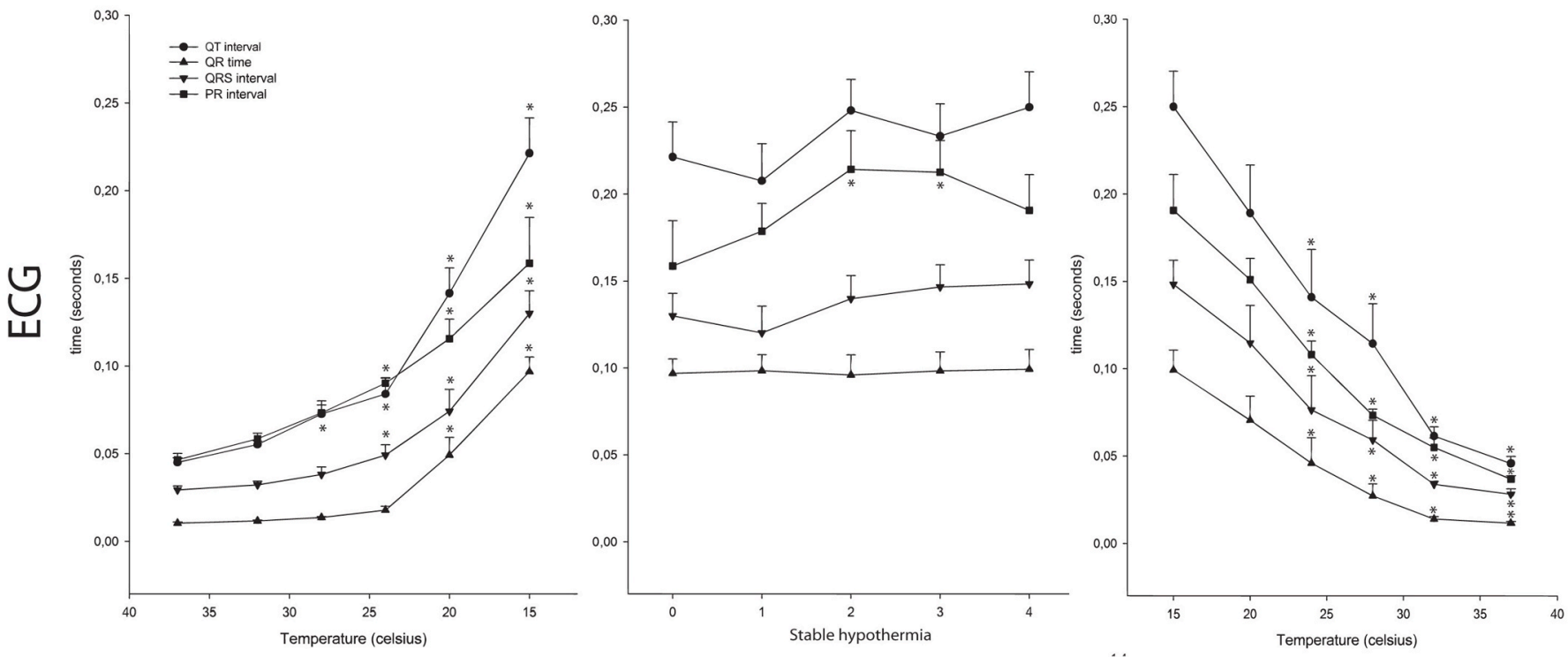

Fig. 1. Changes in electrocardiographic variables during cooling $\left(37^{\circ} \mathrm{C}-15{ }^{\circ} \mathrm{C}\right)$, hypothermia $\left(4 \mathrm{~h}\right.$ at $\left.15{ }^{\circ} \mathrm{C}\right)$ or rewarming $\left(15^{\circ} \mathrm{C}-37^{\circ} \mathrm{C}\right)$. * Significant $(\mathrm{p}<0.05)$ difference from pre-cooling $\left(37^{\circ} \mathrm{C}\right)$, hypothermic $\left(15^{\circ} \mathrm{C}\right)$ or pre-rewarming $\left(15^{\circ} \mathrm{C}\right)$ baseline.
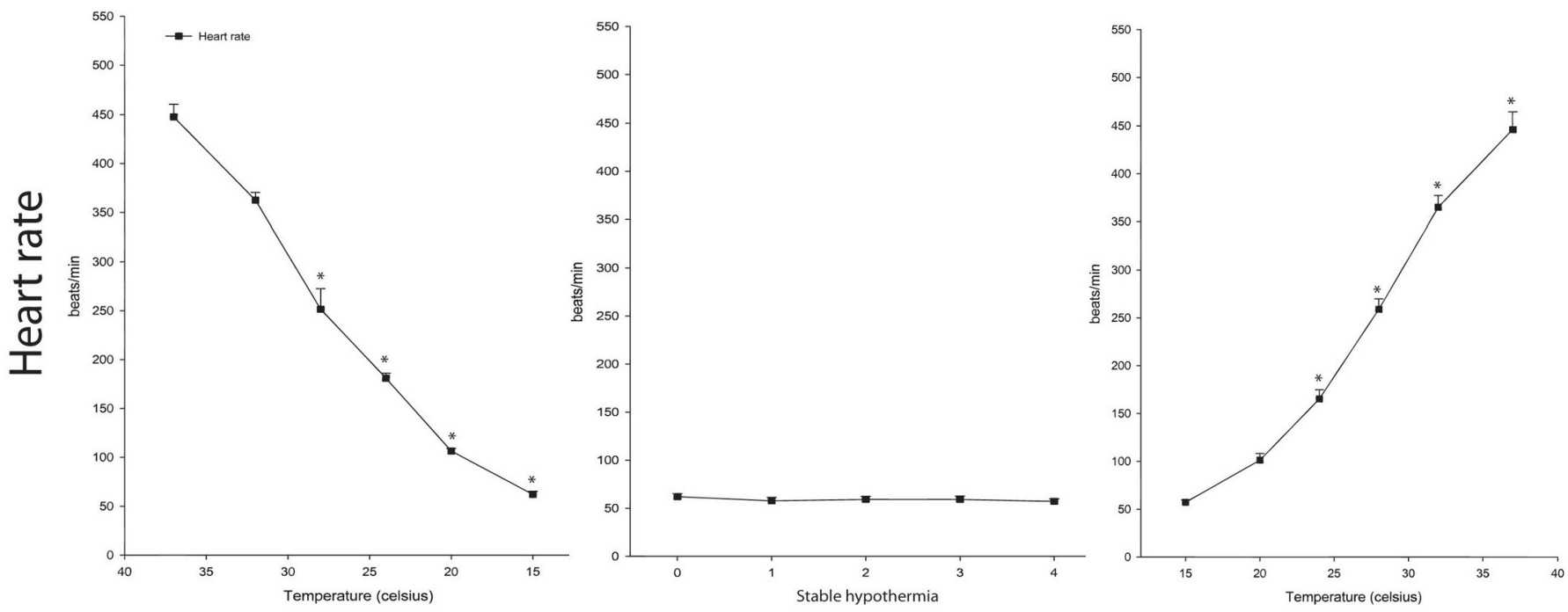

Fig. 2. Changes in heart rate during cooling $\left(37^{\circ} \mathrm{C}-15^{\circ} \mathrm{C}\right)$, hypothermia $\left(4 \mathrm{~h}\right.$ at $\left.15^{\circ} \mathrm{C}\right)$ or rewarming $\left(15^{\circ} \mathrm{C}-37^{\circ} \mathrm{C}\right)$. ${ }^{*}$ Significant $(\mathrm{p}<0.05)$ difference from precooling $\left(37^{\circ} \mathrm{C}\right)$, hypothermic $\left(15^{\circ} \mathrm{C}\right)$ or pre-rewarming $\left(15^{\circ} \mathrm{C}\right)$ baseline.

and animal species that have similar electrophysiology to humans, are therefore of interest [8]. In the present study on rats, the QRS/QTc ratio increased steadily during cooling, remained stable during hypothermia, and was normalised during rewarming. Interestingly, when assessing human ECG-data (Fig. 4) [8], QRS/QTc values were similar to those in rats at normothermia and at severe hypothermia $\left(<24{ }^{\circ} \mathrm{C}\right)$. At these temperatures the QRS/QTc ratios in humans were found to be higher than 0.2 , while initial cooling is associated with a reduction of QRS/QTc values during moderate and higher temperatures of severe hypothermia $\left(32-24{ }^{\circ} \mathrm{C}\right)$. Exposure to such core temperatures is highly associated with occurrence of ventricular arrhythmias in hypothermic patients [8]. This is similar to the relation between temperature and the QRS/QTc ratio values in rabbit hearts, where low QRS/QTc values observed during moderate hypothermia correlated highly with increased VF-risk (VF threshold). In contrast, QRS/QTc values in rat never fell below 0.2, corresponding with absence of spontaneous ventricular arrhythmias in the present experiment.

In a recent study, we found that moderate hypothermia presents a "vulnerable window" for hypothermia-induced VF and cardiac arrest in rabbit hearts [6]. This pro-arrhythmic state was related to slowed cardiac conduction and repolarisation, whilst ventricular/transmural activation remained relatively unaffected, producing an acquired long-QT syndrome [6]. As transmural conduction is gap-junction dependent, we tested the gap-junction uncoupler Heptanol as an anti-arrhythmic measure. After administration, risk for VF was normalised and equal to in normothermic hearts [6].

Hypothermia-induced QT-prolongation is however not species dependent [8] or directly associated with increased risk for VF, as shown in the present rat model, where animals were resistant to hypothermia-induced ventricular arrhythmia despite a five-fold increase in QT-time after cooling to $15^{\circ} \mathrm{C}$. VF-resistance in rats could be ascribed the critical mass hypothesis, saying that fibrillation is only possible in hearts that exceed a critical size. This theory is however challenged by that burst pacing triggered VF in a majority of Langendorff-perfused mouse hearts [26]. Another explanation for this species-dependent difference could be the relative transmural distance across the ventricular 

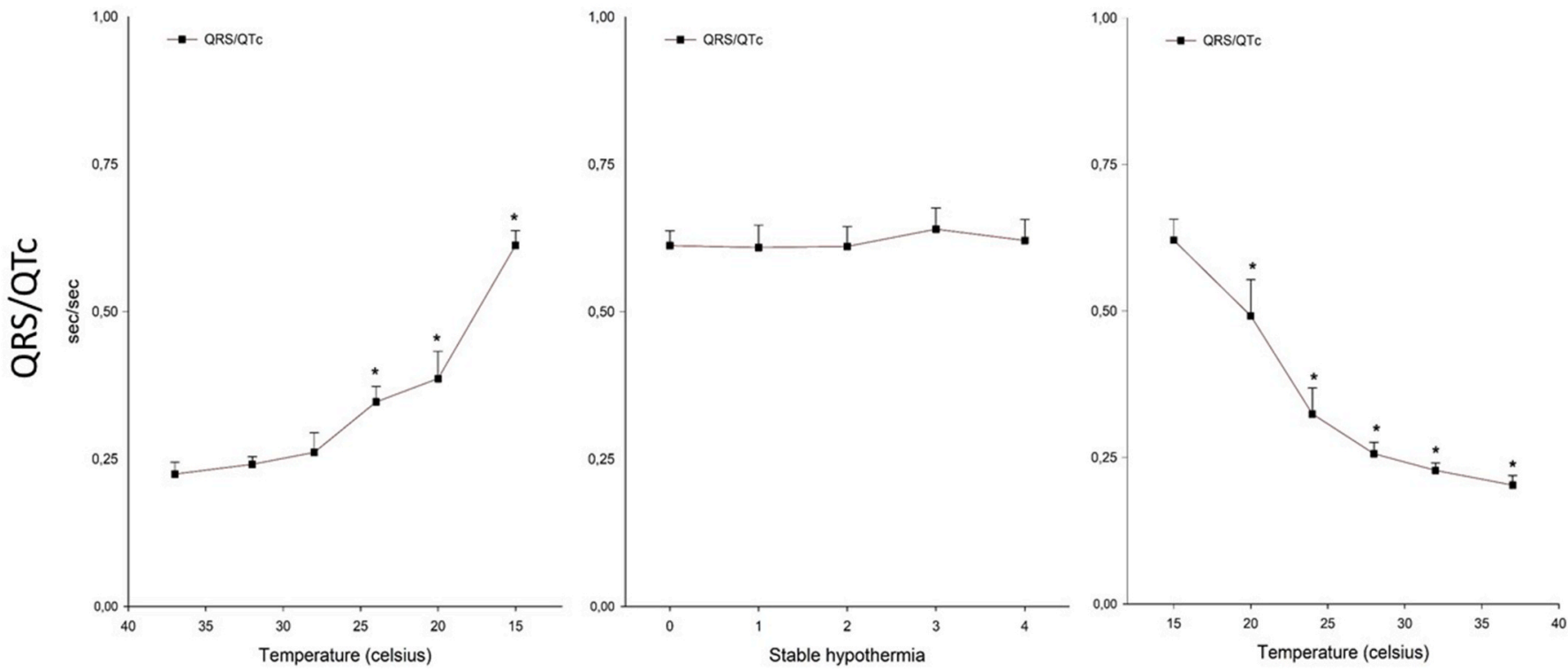

Fig. 3. Changes in QRS/QTc values during cooling $\left(37^{\circ} \mathrm{C}-15^{\circ} \mathrm{C}\right)$, hypothermia $\left(4 \mathrm{~h}\right.$ at $\left.15^{\circ} \mathrm{C}\right)$ or rewarming $\left(15^{\circ} \mathrm{C}-37^{\circ} \mathrm{C}\right)$. * Significant $(\mathrm{p}<0.05)$ difference from pre-cooling $\left(37^{\circ} \mathrm{C}\right)$, hypothermic $\left(15^{\circ} \mathrm{C}\right)$ or pre-rewarming $\left(15^{\circ} \mathrm{C}\right)$ baseline.

\section{Human and rat QRS/QTC}

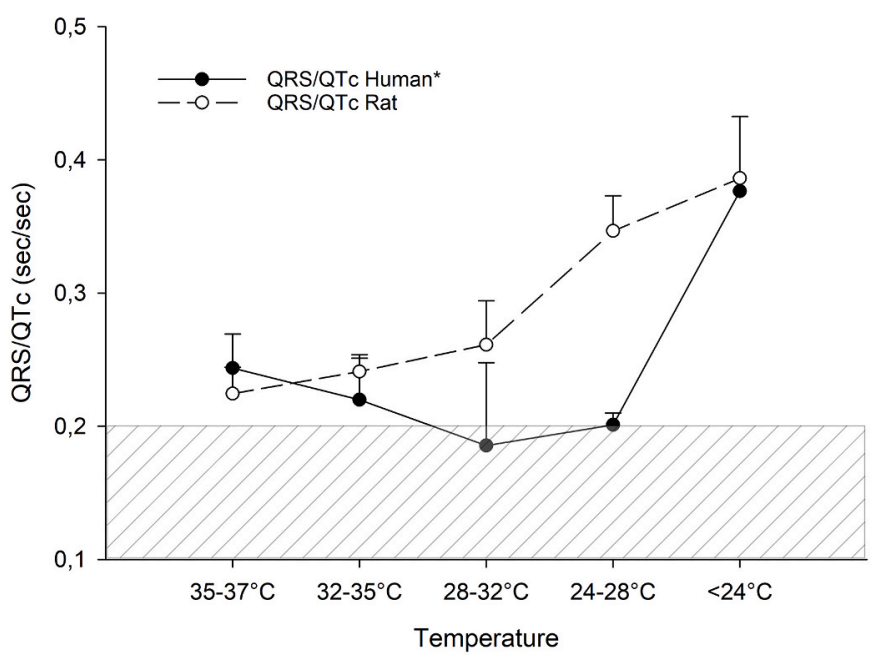

Fig. 4. *QRS/QTc values in rats from the present experiment, compared to recently published human data from our group [4,6]. Rat QRS/QTc values show a steady increase during cooling to severe hypothermia, while values in humans are reduced in moderate hypothermia. Low QRS/QTc values could be associated with increased risk for ventricular fibrillation in hypothermic patients [6].

wall, compared to cardiomyocyte dimensions. Despite small heart size and short transmural distance in rats, compared to both humans and rabbits, cardiomyocyte dimension is comparable across species [21]. Thus, in rats, transmural conduction involves conduction across relatively fewer intercellular elements and therefore is less dependent on gap junction/intercellular resistance and more dependent on intracellular resistance than larger hearts (e.g. rabbits [6] and humans). Therefore, lowered temperature would reduce ventricular/transmural conduction and increase QRS time to a greater extent in rats and small mammals than larger animals. Our data supports this theory, as hypothermia has immediate effects on ventricular activation (QRS time) and repolarisation time (QT), observed through progressive increases in QRS/QTc values during cooling. This indicates that despite prolonged QTc in the rat, the risk of arrythmias is more correctly predicted by a decrease in QRS/QTc ratio. Such reduction is never encountered across the complete range of hypothermia temperatures in rat. This is different from rabbit and human data (Fig. 4) [7]. Accordingly, we speculate that the limited dependence on gap junction activity for transmural conduction is the underlying mechanism for resistance to hypothermia-induced VF in rats, compared to other species like rabbit and hypothermic patients [6-8].

According to our present and previous findings, identifying proarrhythmic activity during hypothermia depends on the ability to detect a heterogenic effect on ventricular/transmural activation, relative to cardiac conduction and repolarisation [6-8]. Therefore, it is not possible to predict the vulnerable window by assessing QT-time alone. Risk for hypothermia-induced arrhythmias rather seems to be associated with an exaggerated shortening of depolarisation timings (QRS) relative to repolarisation time corrected for RR-interval (QTc), namely QRS/QTc. From the present and previous translational studies [6-8], we propose that 0.2 defines the upper limit of the vulnerable window, as measured by QRS/QTc.

\section{Conclusion}

Different from the high incidence of hypothermia-induced ventricular arrhythmias in accidental hypothermia patients, severe hypothermia $\left(4 \mathrm{~h}\right.$ at $\left.15^{\circ} \mathrm{C}\right)$, and rewarming of rats is not associated with increased risk for ventricular fibrillation. This resistance to lethal hypothermiainduced arrhythmias in rats can be predicted by use of a novel on-line ECG-marker; the QRS/QTc ratio.

\section{Sources of funding}

This work was supported by a grant from the Northern Norwegian Health Authority [HNF1337-17]. The funders had no role in study design, data collection and analysis, decision to publish, or preparation of the manuscript.

\section{Availability of data and materials}

The datasets generated during and/or analyzed during the current study are available from the corresponding author on request.

Disclosures of potential conflict of interest.

The authors declare that they have no conflict of interest. 


\section{Research involving animals}

All experiments from which data were used in this study, were undertaken in accordance with guidelines for accommodation and care of animals (article 5 of European Convention for the Protection of Vertebrate Animals used for Experimental and Other Scientific Purposes, Strasbourg, 18.III.1986) and the experimental protocol was approved by the Norwegian Animal Research Authority and conducted accordingly.

\section{Informed consent}

Not applicable.

\section{Acknowledgements}

We thank Togo TV (๔ 2016 DBCLS TogoTV) for kindly allowing us to use their illustration of rat in our graphical abstract.

\section{References}

[1] H. Bjørnstad, D. Jenssen, E. Mortensen, Diltiazem does not increase ventricular fibrillation threshold during hypothermia, Acta Anaesthesiol. Scand. 39 (1995) 659.

[2] T. Darocha, D. Sobczyk, S. Kosinski, A. Jarosz, R. Gałazkowski, K. Nycz, et al., Electrocardiographic changes caused by severe accidental hypothermia, J. Cardiothorac. Vasc. Anesth. 29 (2015) e83.

[3] E.S. Dietrichs, E. Dietrichs, Neuroprotective effects of hypothermia, Tidsskr Nor Legeforen 135 (2015) 1646-1651.

[4] E.S. Dietrichs, B. Håheim, T. Kondratiev, G.C. Sieck, T. Tveita, Cardiovascular effects of levosimendan during rewarming from hypothermia in rat, Cryobiology 69 (3) (2014).

[5] E.S. Dietrichs, B. Håheim, T. Kondratiev, E. Traasdahl, T. Tveita, Effects of hypothermia and rewarming on cardiovascular autonomic control in vivo, J. Appl. Physiol. 124 (2018) 850-859.

[6] E.S. Dietrichs, K. McGlynn, A. Allan, A. Connolly, M. Bishop, F. Burton, et al., Moderate but Not Severe Hypothermia Causes Pro-arrhythmic Changes in Cardiac Electrophysiology, 2020. Cardiovasc Res. [Online ahead of print].

[7] E.S. Dietrichs, T. Tveita, R. Myles, G. Smith, T. Torkjel, R. Myles, et al., A novel ECG-biomarker for cardiac arrest during hypothermia, Scand J Trauma Resusc Emerg Med. Scand J Trauma Resusc Emerg Med. 28 (2020) 27.

[8] E.S. Dietrichs, T. Tveita, G. Smith, Hypothermia and cardiac electrophysiology: a systematic review of clinical and experimental data, Cardiovasc. Res. 115 (2019) 501-509.

[9] F. Ettl, I.A.M. Magnet, W. Weihs, A.-M. Warenits, D. Grassmann, M. Wagner, et al. Establishing a rodent model of ventricular fibrillation cardiac arrest with graded histologic and neurologic damage with different cardiac arrest durations, Shock 50 (2018) 219-225.

[10] M. Gilbert, R. Busund, A. Skagseth, P. A. Nilsen, Solbø JP Resuscitation from accidental hypothermia of 13.7 degrees $\mathrm{C}$ with circulatory arrest, Lancet 355 (2000) 375-376.
[11] Z. Gurabi, I. Koncz, B. Patocskai, V.V. Nesterenko, C. Antzelevitch, Cellular mechanism underlying hypothermia-induced ventricular tachycardia/ventricula fibrillation in the setting of early repolarization and the protective effect of quinidine, cilostazol, and milrinone, Circ Arrhythm Electrophysiol 7 (2014) $134-142$.

[12] Y.-C. Hsieh, W.-H. Hsieh, C.-H. Li, Y.-C. Liao, J.-C. Lin, C.-J. Weng, et al, Ventricular divergence correlates with epicardial wavebreaks and predicts ventricular arrhythmia in isolated rabbit hearts during therapeutic hypothermia, in: M. Zarzoso (Ed.), PLoS One vol. 15, 2020 e0228818.

[13] Y.-C. Hsieh, S.-F. Lin, T.-C. Lin, C.-T. Ting, T.-J. Wu, Therapeutic hypothermia $\left(30^{\circ} \mathrm{C}\right)$ enhances arrhythmogenic substrates, including spatially discordant alternans, and facilitates pacing-induced ventricular fibrillation in isolated rabbit hearts, Circ. J. 73 (2009) 2214-2222.

[14] L. Lamoureux, J. Radhakrishnan, R.J. Gazmuri, A rat model of ventricular fibrillation and resuscitation by conventional closed-chest technique, JoVE (98) (2015) 52413.

[15] H.R. Lu, G.X. Yan, D.J. Gallacher, A new biomarker-index of cardiac electrophysiological balance (iCEB)-plays an important role in drug-induced cardiac arrhythmias: beyond QT-prolongation and Torsades de Pointes (TdPs), J. Pharmacol. Toxicol. Methods 68 (2) (2013) 250-259.

[16] S.A. Mirzoyev, C.J. McLeod, T.J. Bunch, M.R. Bell, R.D. White, Hypokalemia during the cooling phase of therapeutic hypothermia and its impact on arrhythmogenesis, Resuscitation 81 (2010) 1632-1636.

[17] R.W. Neumar, M. Shuster, C.W. Callaway, L.M. Gent, D.L. Atkins, F. Bhanji, et al., American Heart Association guidelines update for CPR and emergency cardiovascular care, Circulation 132 (2015) 315-367.

[18] M. Okada, The cardiac rhythm in accidental hypothermia, J. Electrocardiol. 17 (1984) 123.

[19] J.S. Piktel, D. Jeyaraj, T.H. Said, D.S. Rosenbaum, L.D. Wilson, Enhanced dispersion of repolarization explains increased arrhythmogenesis in severe versus therapeutic hypothermia, Circ Arrhythm Electrophysiol 4 (2010) 79-86.

[20] P. Salinas, E. Lopez-de-Sa, L. Pena-Conde, A. Viana-Tejedor, J.R. Rey-Blas, E. Armada et al. Electrocardiographic changes during induced therapeutic hypothermia in comatose survivors after cardiac arrest, World J. Cardiol. 7 (2015) 423.

[21] H. Satoh, L.M.D. Delbridge, L.A. Blatter, D.M. Bers, Surface:volume relationship in cardiac myocytes studied with confocal microscopy and membrane capacitance measurements: species-dependence and developmental effects, Biophys. J. 70 (1996) 1494-1504.

[22] J.H. Thomsen, J. Kjaergaard, C. Graff, S. Pehrson, D. Erlinge, M. Wanscher, et al, Ventricular ectopic burden in comatose survivors of out-of-hospital cardiac arrest treated with targeted temperature management at $33^{\circ} \mathrm{C}$ and $36^{\circ} \mathrm{C}$, Resuscitation 102 (2016) 98-104.

[23] A. Truhlár̆, C.D. Deakin, J. Soar, G.E.A. Khalifa, A. Alfonzo, J.J.L.M. Bierens, et al, European resuscitation council guidelines for resuscitation 2015: section 4. Cardiac arrest in special circumstances, Resuscitation 95 (2015) 148-201.

[24] T. Tveita, M. Skandfer, H. Refsum, K. Ytrehus, Experimental hypothermia and rewarming: changes in mechanical function and metabolism of rat hearts, J Appl Physiol. Am Physiological Soc. 80 (1996) 291-297.

[25] M.R. Ujhelyi, J.J. Sims, S.A. Dubin, J. Vender, A.W. Miller, Defibrillation energy requirements and electrical heterogeneity during total body hypothermia, Crit. Care Med. 29 (2001) 1006.

[26] D. Vaidya, G.E. Morley, F.H. Samie, J. Jalife, Reentry and fibrillation in the mouse heart. A challenge to the critical mass hypothesis, Circ. Res. 23 (2) (1999) 174-181, 85. 